\title{
Stabilization of Cascaded Two-Port Networked Systems Against Nonlinear Perturbations
}

\author{
Di Zhao, Sei Zhen Khong, and Li Qiu
}

\begin{abstract}
A networked control system (NCS) consisting of cascaded two-port communication channels between the plant and controller is modeled and analyzed. Towards this end, the robust stability of a standard closed-loop system in the presence of conelike perturbations on the system graphs is investigated. The underlying geometric insights are then exploited to analyze the two-port NCS. It is shown that the robust stability of the two-port NCS can be guaranteed when the nonlinear uncertainties in the transmission matrices are sufficiently small in norm. The stability condition, given in the form of "arcsin" of the uncertainty bounds, is both necessary and sufficient.
\end{abstract}

\section{INTRODUCTION}

Feedback is widely used for handling modeling uncertainties in the area of systems and control. Within a feedback loop, communication between the plant and controller plays an important role in that the achieved control performance and robustness heavily rely on the quality of communication. In practice, communication can never be ideal due to the presence of channel distortions and interferences. In this study, we analyze the robust stability of a feedback system involving bidirectional uncertain communication modeled by cascaded two-port networks.

Most control systems can be regarded as structured networks with signals transmitted through channels powered by various devices, such as sensors or satellites. A networked control system (NCS) differs from a standard closed-loop system in that the information is exchanged through a communication network [1]. The presence of such a network may introduce disturbances to a control system and hence significantly compromise its performance.

In this study, we introduce an NCS model, extending the standard linear time-invariant (LTI) closed-loop system (Fig. 11) to the feedback system with cascaded two-port connections (Fig. 2). We assume that the controller and plant are LTI while the two-port networks involve nonlinear perturbations on their transmission matrices. In terms of communication uncertainties, we model the transmission matrices as $\boldsymbol{T}=\boldsymbol{I}+\boldsymbol{\Delta}$, where $\boldsymbol{\Delta}$ is a bounded nonlinear operator. Our formulation of robust stabilization problem is mainly

\footnotetext{
*This work was supported in part by the Research Grants Council of Hong Kong Special Administrative Region, China, under projects 16201115 and $\mathrm{T} 23-701 / 14 \mathrm{~N}$

D. Zhao and L. Qiu are with the Department of Electronic \& Computer Engineering, The Hong Kong University of Science and Technology, Clear Water Bay, Kowloon, Hong Kong, China. dzhaoaa@ust.hk, eeqiudece.ust.hk

S. Z. Khong is with the Institute for Mathematics and its Applications, University of Minnesota, Minneapolis, MN 55455, USA. szkhong@umn. edu
}

motivated by the application scenario of stabilizing a feedback system where the plant and controller do not possess an ideal communication environment and their input-output signals can only be sent through communication networks with several relays, as in, for example, teleoperation systems [2], satellite networks [3], wireless sensor networks [4] and so on. Moreover, each sub-system between two neighbouring relays, representing a communication channel, may involve not only multiplicative distortions on the transmitted signal itself but also additive interferences caused by the signal in the reverse direction, which is usually encountered in a bidirectional wireless network subject to channel fading or under malicious attacks [5].

Two-port networks are not a new concept and have been studied for decades for different purposes. Historically, twoport networks were first introduced in electrical circuits theory [6]. Later on they were utilized to represent LTI systems in the so-called chain-scattering formalism [7], which is essentially a two-port network. Such representations have also been used for studying feedback robustness from the perspective of the $\nu$-gap metric [8]. Recently, approaches based on the two-port network to modeling communication channels in a networked feedback system is studied in [9] and [10]. There, uncertain two-port connections are used to introduce channel uncertainties, based on which we propose our cascaded two-port communication model with nonlinear perturbations in this paper.

One of the contributions of our study is a clean result for analyzing the stability of a feedback system with multiple sources of uncertainties. A general approach to robust stabilization of LTI systems with structured uncertainties is $\mu$ analysis, which is known to be computationally intractable in general in the presence of multiple uncertainties [11]. Furthermore, the two-port uncertainties in this study are nonlinear, which bring in an additional obstacle. To overcome these difficulties, we take advantage of the special two-port structures and make use of geometric insights on system stability via an input-output approach. By generalizing the "arcsin" theorem in [12] for a standard closed-loop system, we are able to give a concise necessary and sufficient robust stability condition for the two-port NCS. Moreover, the stability condition is scalable and computationally friendly, in the sense that when the topology of the two-port NCS is changed, the stability condition can be efficiently updated based only on the modified components. In terms of designing an optimal controller, it suffices to solve an $\mathcal{H}_{\infty}$ optimization problem, which is mathematically tractable.

It is worth noting that there exist previous works on 


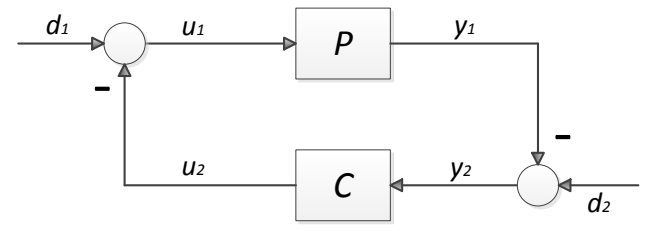

Fig. 1: A Standard Closed-Loop System

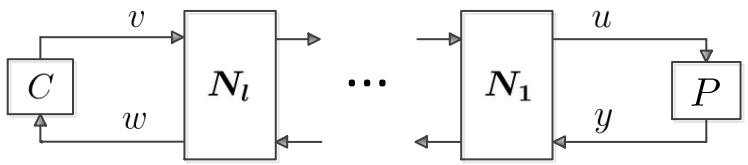

Fig. 2: Communication Channels Modeled by Cascaded Two-port Networks

robust stabilization of NCSs with special architectures and various uncertainty descriptions. For example, [2] considers teleoperation of robots through two-port communication networks with time-delay, [13] considers a plant with parametric uncertainties over networks subject to packet loss, [14] considers a plant with polytopic uncertainties in its coefficients over a communication channel subject to fadings and so on. The differences of our work from the previous ones are that our channel model characterizes bi-directional communication involving both distortions and interferences and these uncertainties may be nonlinear.

The rest of the paper is organized as follows. First in Section [II] we define open-loop stability, closed-loop wellposedness and stability, system uncertainties and some related properties. Then in Section III] we give a robust stability condition for a closed-loop system with conelike uncertainty descriptions. Thereafter in Section IV] we extend the results on robust stability to cascaded two-port networks. In Section V] we conclude this study and summarize our contributions.

\section{PReliminaries}

\section{A. Open-loop Stability}

Let $\mathcal{H}_{2}^{n}:=\left\{f:\left.[0, \infty) \rightarrow \mathbb{R}^{n}\left|\|f\|_{2}^{2}:=\int_{0}^{\infty}\right| f(t)\right|^{2} d t<\right.$ $\infty\}$, where $|\cdot|$ denotes the Euclidean norm. Let $\mathcal{R} \mathcal{H}_{\infty}$ consist of all the real rational members of $\mathcal{H}_{\infty}$, the Hardy $\infty$-space of functions that are holomorphic on the right-half complex plane.

Denote the time truncation operator at time $\tau \in[0, \infty)$ as $\boldsymbol{T}_{\tau}$, such that for $u(t) \in \mathcal{H}_{2}$,

$$
\left(\boldsymbol{T}_{\tau} u\right)(t)= \begin{cases}u(t), & 0 \leq t<\tau \\ 0, & \text { Otherwise }\end{cases}
$$

A nonlinear system is represented by an operator $\boldsymbol{P}$ : $\operatorname{dom}(\boldsymbol{P}) \subset \mathcal{H}_{2} \mapsto \mathcal{H}_{2}$ with domain $\operatorname{dom}(\boldsymbol{P})=\{u \in$ $\left.\mathcal{H}_{2} \mid \boldsymbol{P} u \in \mathcal{H}_{2}\right\}$. We denote its image as $\operatorname{img}(\boldsymbol{P})$. A physical system should additionally be causal, which is defined as follows [15].
Definition 1. A nonlinear system $\boldsymbol{P}: \operatorname{dom}(\boldsymbol{P}) \subset \mathcal{H}_{2} \mapsto \mathcal{H}_{2}$ is said to be causal if for every $\tau \in[0, \infty)$ and $u_{1}, u_{2} \in$ $\operatorname{dom}(\boldsymbol{P})$,

$$
\boldsymbol{T}_{\tau} u_{1}=\boldsymbol{T}_{\tau} u_{2} \Rightarrow \boldsymbol{T}_{\tau} \boldsymbol{P} u_{1}=\boldsymbol{T}_{\tau} \boldsymbol{P} u_{2}
$$

We assume $\boldsymbol{P} 0=0$ throughout this study, which means every nonlinear system we consider has zero output whenever the input is zero. The finite-gain stability of a system is defined as follows [16].

Definition 2. A causal nonlinear operator (system) $\boldsymbol{P}$ is said to be (finite-gain) stable if $\operatorname{dom}(\boldsymbol{P})=\mathcal{H}_{2}$ and its operator norm is bounded, that is

$$
\|\boldsymbol{P}\|:=\sup _{0 \neq x \in \mathcal{H}_{2}} \frac{\|\boldsymbol{P} x\|_{2}}{\|x\|_{2}}<\infty .
$$

\section{B. Closed-loop Stability}

We consider a standard closed-loop system in Fig. 1 with plant $\boldsymbol{P}: \operatorname{dom}(\boldsymbol{P}) \subset \mathcal{H}_{2}^{p} \mapsto \mathcal{H}_{2}^{m}$ and controller $\boldsymbol{C}: \operatorname{dom}(\boldsymbol{C}) \subset \mathcal{H}_{2}^{m} \mapsto \mathcal{H}_{2}^{p}$. In the following, the superscripts of $\mathcal{H}_{2}^{m}$ and $\mathcal{H}_{2}^{p}$ will be omitted for notational simplicity.

The graph of $\boldsymbol{P}$ is defined as

$$
\mathcal{G}_{\boldsymbol{P}}=\left[\begin{array}{l}
\boldsymbol{I} \\
\boldsymbol{P}
\end{array}\right] \operatorname{dom}(\boldsymbol{P})
$$

and similarly the inverse graph of $C$ is defined as

$$
\mathcal{G}_{C}^{\prime}=\left[\begin{array}{l}
\boldsymbol{C} \\
\boldsymbol{I}
\end{array}\right] \operatorname{dom}(\boldsymbol{C})
$$

both of which are assumed to be closed in this study.

It can be seen in [15]-[17] that various versions of feedback well-posedness may be assumed based on different signal spaces and causality requirements. In this study, we adopt the well-posedness definition from [17] without appealing to extended spaces, by contrast to, for example, [15], [16].

Definition 3. The closed-loop system $[\boldsymbol{P}, \boldsymbol{C}]$ is said to be well-posed if

$$
\begin{aligned}
& \boldsymbol{F}_{\boldsymbol{P}, \boldsymbol{C}}: \operatorname{dom}(\boldsymbol{P}) \times \operatorname{dom}(\boldsymbol{C}) \mapsto \mathcal{H}_{2} \\
&:=\left[\begin{array}{l}
u_{1} \\
y_{2}
\end{array}\right] \mapsto\left[\begin{array}{l}
d_{1} \\
d_{2}
\end{array}\right]=\left[\begin{array}{ll}
\boldsymbol{I} & \boldsymbol{C} \\
\boldsymbol{P} & \boldsymbol{I}
\end{array}\right]\left[\begin{array}{l}
u_{1} \\
y_{2}
\end{array}\right]
\end{aligned}
$$

is causally invertible on $\operatorname{img}\left(\boldsymbol{F}_{\boldsymbol{P}, \boldsymbol{C}}\right)$.

Correspondingly, the stability of the closed-loop system is defined as follows:

Definition 4. A well-posed closed-loop system $[\boldsymbol{P}, \boldsymbol{C}]$ is (finite-gain) stable if $\boldsymbol{F}_{\boldsymbol{P}, \boldsymbol{C}}$ is surjective and $\boldsymbol{F}_{\boldsymbol{P}, \boldsymbol{C}}^{-1}$ is finitegain stable.

When $\boldsymbol{F}_{P, C}$ is surjective, the parallel projection operators [18] along $\mathcal{G}_{P}$ and $\mathcal{G}_{C}^{\prime}, \Pi_{\mathcal{G}_{P} / / \mathcal{G}_{C}^{\prime}}$ and $\Pi_{\mathcal{G}_{C}^{\prime} / / \mathcal{G}_{P}}$, can be defined respectively as

$$
\begin{aligned}
\Pi_{\mathcal{G}_{\boldsymbol{P}} / / \mathcal{G}_{C}^{\prime}}: & {\left[\begin{array}{l}
d_{1} \\
d_{2}
\end{array}\right] \in \mathcal{H}_{2} \mapsto\left[\begin{array}{l}
u_{1} \\
y_{1}
\end{array}\right] \in \mathcal{G}_{\boldsymbol{P}} } \\
& =\left[\begin{array}{cc}
\boldsymbol{I} & 0 \\
0 & -\boldsymbol{I}
\end{array}\right] \boldsymbol{F}_{\boldsymbol{P}, \boldsymbol{C}}^{-1}+\left[\begin{array}{cc}
0 & 0 \\
0 & \boldsymbol{I}
\end{array}\right],
\end{aligned}
$$




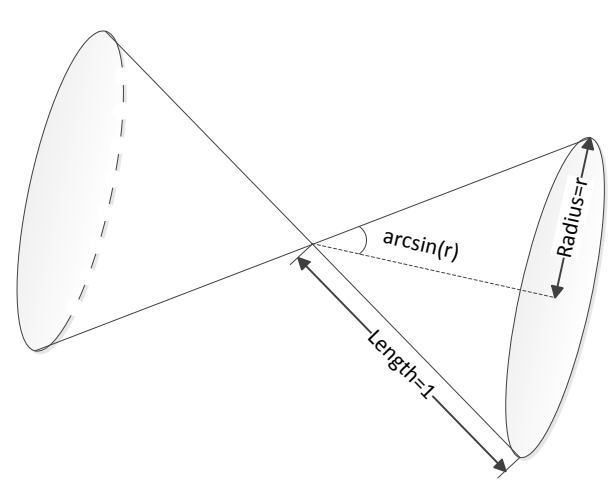

Fig. 3: The conelike neighborhood in $\mathbb{R}^{3}$

$$
\begin{aligned}
\Pi_{\mathcal{G}_{\boldsymbol{C}}^{\prime} / \mathcal{G}_{\boldsymbol{P}}}: & {\left[\begin{array}{l}
d_{1} \\
d_{2}
\end{array}\right] \in \mathcal{H}_{2} \mapsto\left[\begin{array}{l}
u_{2} \\
y_{2}
\end{array}\right] \in \mathcal{G}_{\boldsymbol{C}}^{\prime} } \\
& =\left[\begin{array}{cc}
-\boldsymbol{I} & 0 \\
0 & \boldsymbol{I}
\end{array}\right] \boldsymbol{F}_{\boldsymbol{P}, \boldsymbol{C}}^{-1}+\left[\begin{array}{ll}
\boldsymbol{I} & 0 \\
0 & 0
\end{array}\right] .
\end{aligned}
$$

It follows that every $w \in \mathcal{H}_{2}$ has a unique decomposition as $w=m+n$ with $m=\Pi_{\mathcal{G}_{P} / / \mathcal{G}_{C}^{\prime}} w \in \mathcal{G}_{P}$ and $n=$ $\Pi_{\mathcal{G}_{C}^{\prime} / / \mathcal{G}_{P}} w \in \mathcal{G}_{C}^{\prime}$.

The next proposition bridges the finite-gain stability and the boundedness of parallel projections [18].

Proposition 1. A well-posed closed-loop system $[\boldsymbol{P}, \boldsymbol{C}]$ is stable if and only if $\boldsymbol{F}_{\boldsymbol{P}, \boldsymbol{C}}$ is surjective and $\Pi_{\mathcal{G}_{P} / / \mathcal{G}_{C}^{\prime}}$ or $\Pi_{\mathcal{G}_{C}^{\prime} / / \mathcal{G}_{P}}$ is finite-gain stable.

For a finite-gain stable closed-loop system $[\boldsymbol{P}, \boldsymbol{C}]$, its stability margin is defined as $b_{P, C}:=\left\|\Pi_{\mathcal{G}_{P} / / \mathcal{G}_{C}^{\prime}}\right\|^{-1}$. It is shown in [18] that if either $\boldsymbol{P}$ or $\boldsymbol{C}$ is linear, then $b_{P, C}=b_{C, P}$.

\section{System Uncertainties}

A well-known method to introduce system uncertainties is through various variants of the "gap" or "aperture" between system graphs [19]. In this study, before characterizing the uncertainties in two-port networks, we introduce a useful notion of neighborhood of a certain nominal system's graph, which may serve as its uncertainty set. Let $\mathcal{M}$ be a manifold in $\mathcal{H}_{2}$. Define the conelike neighborhood of $\mathcal{M}$ as

$$
\mathcal{S}(\mathcal{M}, r)=\left\{n \in \mathcal{H}_{2}: \inf _{0 \neq m \in \mathcal{M}} \frac{\|n-m\|_{2}}{\|m\|_{2}} \leq r\right\} \cup\{0\} .
$$

If $\mathcal{M}$ is a one-dimensional subspace in $\mathbb{R}^{3}$, the set $\mathcal{S}(\mathcal{M}, r)$ is simply a right circular double cone as shown in Fig. 3. In case of one-dimensional subspace in $\mathbb{R}^{2}$, the set can be interpreted as doubly sector-bounded area [20]. If $\mathcal{M}$ is the graph of certain linear system, it "resembles" a closed double cone in the space of $\mathcal{H}_{2}$, which provides us some geometric intuitions on the system uncertainties.

Based on the Hilbert space structure of $\mathcal{H}_{2}$, let $\theta(x, y)$ denote the acute angle between $x, y \in \mathcal{H}_{2} \backslash\{0\}$ and $\theta(x, y)=\infty$ if either of $x, y$ is zero almost everywhere for convenience.
Given $r \in(0,1)$ and a closed conelike neighboring set $\mathcal{M} \subset \mathcal{H}_{2}$, we have the following useful properties:

Property 1. Let $n \in \mathcal{H}_{2} \backslash\{0\}$. Then $n \in \mathcal{S}(\mathcal{M}, r)$ if and only if $\alpha n \in \mathcal{S}(\mathcal{M}, r)$ for every $\alpha \in \mathbb{R}$;

Property 2. $\mathcal{S}(\mathcal{M}, r)=\left\{n \in \mathcal{H}_{2}: \inf _{m \in \mathcal{M}} \theta(m, n) \leq\right.$ $\arcsin r\} \cup\{0\}$.

Another related neighboring set is defined as follows:

$$
\tilde{\mathcal{S}}(\mathcal{M}, r):=\left\{n \in \mathcal{H}_{2} \backslash\{0\}: \inf _{m \in \mathcal{M}} \frac{\|n-m\|_{2}}{\|n\|_{2}} \leq r\right\} \cup\{0\} .
$$

Property 3. $\mathcal{S}(\mathcal{M}, r)=\tilde{\mathcal{S}}(\mathcal{M}, r)$.

The proofs for the above properties are in Appendix [ which follow from the definition of conelike neighborhoods directly.

Remark 1. In general, $\tilde{\mathcal{S}}(\mathcal{M}, r) \neq \mathcal{S}(\mathcal{M}, r)$ for arbitrary manifold $\mathcal{M}$ in $\mathcal{H}_{2}$.

One benefit of defining uncertainties as above is that we can examine the intersection of two cones simply by studying the angles between two lines from each of them respectively. Moreover, the intersection of the graphs may reflect the instability of a closed-loop system, as is detailed in the next section.

\section{Feedback Interconnections With Conelike UNCERTAINTIES}

Given a (possibly unstable) LTI nominal closed-loop system $[P, C]$ with open-loop system graphs $\mathcal{G}_{P}$ and $\mathcal{G}_{C}^{\prime}$, we have the following result concerning its robust stability, whose proof is in Appendix II]

Proposition 2. Given $r_{p}, r_{c} \in(0,1)$, the perturbed system $\left[\boldsymbol{P}_{1}, \boldsymbol{C}_{1}\right]$ is stable for all $\mathcal{G}_{\boldsymbol{P}_{1}} \subset \mathcal{S}\left(\mathcal{G}_{P}, r_{p}\right), \mathcal{G}_{\boldsymbol{C}_{1}}^{\prime} \subset$ $\mathcal{S}\left(\mathcal{G}_{C}^{\prime}, r_{c}\right)$ such that $\boldsymbol{F}_{\boldsymbol{P}_{1}, \boldsymbol{C}_{1}}$ is surjective if and only if

$$
\mathcal{S}\left(\mathcal{G}_{P}, r_{p}\right) \cap \mathcal{S}\left(\mathcal{G}_{C}^{\prime}, r_{c}\right)=\{0\} .
$$

It is known that a standard well-posed LTI closed-loop system $[P, C]$ is stable if and only if $\mathcal{G}_{P} \oplus \mathcal{G}_{C}^{\prime}=\mathcal{H}_{2} \times \mathcal{H}_{2}$. As there is no subspace representation for the graph of a nonlinear system, Proposition 2 generalizes the geometric insight of complementarity of subspaces. Building on that, we have the following robust stability condition, which extends the "arcsin" inequality condition in [12] and [21].

Theorem 1. Assume the LTI nominal closed-loop system $[P, C]$ is stable. The following statements are equivalent:

1) The perturbed system $\left[\boldsymbol{P}_{1}, \boldsymbol{C}_{1}\right]$ is stable for all $\mathcal{G}_{\boldsymbol{P}_{1}} \subset$ $\mathcal{S}\left(\mathcal{G}_{P}, r_{p}\right), \mathcal{G}_{C_{1}}^{\prime} \subset \mathcal{S}\left(\mathcal{G}_{C}^{\prime}, r_{c}\right)$ such that $\boldsymbol{F}_{\boldsymbol{P}_{1}, C_{1}}$ is surjective;

2) $\mathcal{S}\left(\mathcal{G}_{P}, r_{p}\right) \cap \mathcal{S}\left(\mathcal{G}_{C}^{\prime}, r_{c}\right)=\{0\}$;

3) $\arcsin r_{p}+\arcsin r_{c}<\arcsin b_{P, C}$.

Proof. The equivalence between 1) and 2) has been established in Proposition 2. The direction 1) $\Rightarrow 3$ ) follows from the "arcsin" theorem in [12] for LTI systems by noting that standard the gap metric balls $\mathcal{B}\left(\mathcal{G}_{P}, r_{p}\right)$ and $\mathcal{B}\left(\mathcal{G}_{P}, r_{c}\right)$ 
are contained in the conelike sets $\mathcal{S}\left(\mathcal{G}_{P}, r_{p}\right)$ and $\mathcal{S}\left(\mathcal{G}_{C}^{\prime}, r_{c}\right)$, respectively.

Next we show 3) $\Rightarrow 2$ ). First note that $b_{P, C}=$ $\inf _{m \in \mathcal{G}_{P}, n \in \mathcal{G}_{C}^{\prime}} \sin \theta(m, n)$ from [12]. Given any $m_{1} \in$ $\mathcal{S}\left(\mathcal{G}_{P}, r_{p}\right)$ and $n_{1} \in \mathcal{S}\left(\mathcal{G}_{C}^{\prime}, r_{c}\right)$, the triangle inequality for $\theta(\cdot, \cdot)$ gives

$$
\theta\left(m_{1}, n_{1}\right) \geq \theta(m, n)-\theta\left(m, m_{1}\right)-\theta\left(n, n_{1}\right)
$$

for any $m \in \mathcal{G}_{P}$ and $n \in \mathcal{G}_{C}^{\prime}$.

It follows directly that $\inf _{m \in \mathcal{G}_{P}} \theta\left(m, m_{1}\right) \leq \arcsin r_{p}$ and $\inf _{n \in \mathcal{G}_{C}^{\prime}} \theta\left(n, n_{1}\right) \leq \arcsin r_{c}$ from Property 2 Let $\bar{m} \in$ $\arg \min _{m \in \mathcal{G}_{P}} \theta\left(m, m_{1}\right)$ and $\bar{n} \in \arg \min _{n \in \mathcal{G}_{C}^{\prime}} \theta\left(n, n_{1}\right)$, in which the minimums are achieved due to the closedness of the system graphs. Then inequality (3) implies that

$$
\begin{aligned}
\theta\left(m_{1}, n_{1}\right) & \geq \theta(\bar{m}, \bar{n})-\theta\left(\bar{m}, m_{1}\right)-\theta\left(\bar{n}, n_{1}\right) \\
& \geq \theta(\bar{m}, \bar{n})-\arcsin r_{p}-\arcsin r_{c} \\
& \geq \arcsin b_{P, C}-\arcsin r_{p}-\arcsin r_{c}=: \epsilon>0 .
\end{aligned}
$$

Hence it holds that

$$
\inf _{m_{1} \in \mathcal{S}\left(\mathcal{G}_{P}, r_{p}\right), n_{1} \in \mathcal{S}\left(\mathcal{G}_{C}^{\prime}, r_{c}\right)} \arcsin \theta\left(m_{1}, n_{1}\right) \geq \epsilon>0,
$$

which implies

$$
\mathcal{S}\left(\mathcal{G}_{P}, r_{p}\right) \cap \mathcal{S}\left(\mathcal{G}_{C}^{\prime}, r_{c}\right)=\{0\},
$$

as required.

A short summary to the above results follows. A certain robust stability condition is derived while allowing simultaneous perturbations on the plant and controller, in the expression of an "arcsin" inequality. The uncertainties are measured with conelike neighborhoods. It is worth noting that for nonlinear systems, $\delta$-type gaps and $\gamma$-type gaps can be used to characterize the set of all neighboring system graphs within some radius [19], which defines a set of manifolds. On the other hand, a conelike neighborhood simply gathers all input-output pairs of a certain distance from the center, which forms a manifold itself. The advantage of focusing on input-output pairs instead of system graphs arises in the case where only partial information about the graph of a nonlinear system is available, say in the form of some measured input-output data set, which may not be sufficient for the purpose of computing the gap-distance, rendering standard gap-type stability conditions inapplicable. On the contrary, if the uncertainties are measured with respect to the available input-output pairs, it is likely that the limited measured data are sufficient to give a good approximation of these uncertainties. To verify whether a partially known perturbed system lies within a conelike neighborhood, it suffices to check every available input-output pair.

\section{NeTworked Robust Stabilization with CASCAded Nonlinear UnCERTainties}

\section{A. Two-Port Networks as Communication Channels}

The use of two-port networks as a model of communication channels is adopted from [9], [10]. Two-port networks were first introduced and investigated in electrical circuits

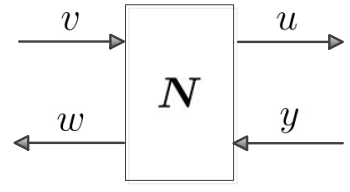

(a) A Single Two-Port Network

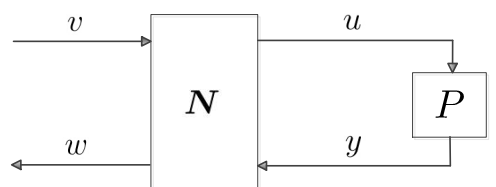

(b) One-stage Two-Port Connection

Fig. 4: Two-Port Networks: an Illustration

theory [6]. The network $\boldsymbol{N}$ in Fig. 4a has two external ports, with one port composed of $v, w$ and the other of $u, y$, and is called a two-port network. A two-port network $\boldsymbol{N}$ may have various representations, out of which we choose the transmission type to model a communication channel. Define the transmission matrix $T$ as

$$
\boldsymbol{T}=\left[\begin{array}{ll}
\boldsymbol{T}_{11} & \boldsymbol{T}_{12} \\
\boldsymbol{T}_{21} & \boldsymbol{T}_{22}
\end{array}\right] \text { and }\left[\begin{array}{c}
v \\
w
\end{array}\right]=\boldsymbol{T}\left[\begin{array}{l}
u \\
y
\end{array}\right] .
$$

When the communication channel is perfect, i.e., communication takes place without distortion or interference, the transmission matrix is simply

$$
\boldsymbol{T}=\left[\begin{array}{cc}
\boldsymbol{I}_{m} & 0 \\
0 & \boldsymbol{I}_{p}
\end{array}\right] .
$$

If the bidirectional channel admits both distortions and interferences, we can let the transmission matrix take the form

$$
\boldsymbol{T}=\boldsymbol{I}+\boldsymbol{\Delta}=\left[\begin{array}{cc}
\boldsymbol{I}_{m}+\boldsymbol{\Delta}_{\doteqdot} & \boldsymbol{\Delta}_{-} \\
\boldsymbol{\Delta}_{+} & \boldsymbol{I}_{p}+\boldsymbol{\Delta}_{\times}
\end{array}\right],
$$

where $\boldsymbol{I}: \mathcal{H}_{2} \mapsto \mathcal{H}_{2}$ is the identity operator and

$$
\boldsymbol{\Delta}=\left[\begin{array}{cc}
\boldsymbol{\Delta}_{\div} & \boldsymbol{\Delta}_{-} \\
\boldsymbol{\Delta}_{+} & \boldsymbol{\Delta}_{\times}
\end{array}\right]: \mathcal{H}_{2} \mapsto \mathcal{H}_{2}
$$

satisfies $\|\boldsymbol{\Delta}\| \leq r<1$, which ensures that $\boldsymbol{T}$ is stably invertible. The four-block matrix $\Delta$ is called the uncertainty quartet.

\section{B. Graph Analysis on Cascaded Two-Port NCS}

It is well known that graphs symbols can be defined for finite-dimensional LTI systems [11]. For every LTI system with transfer function $P$, it admits a right coprime factorization $P=N M^{-1}$ satisfying $X M+Y N=I$, where $M, N, X, Y \in \mathcal{R} \mathcal{H}_{\infty}$. The graph symbol is defined as

$$
\left[\begin{array}{c}
M \\
N
\end{array}\right], \text { whereby } \mathcal{G}_{P}=\left[\begin{array}{c}
M \\
N
\end{array}\right] \mathcal{H}_{2} ;
$$

see [19, Proposition 1.33]. 


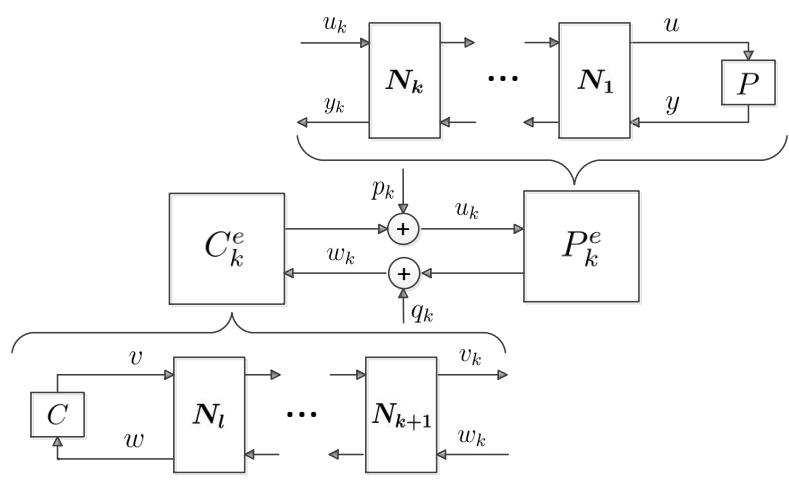

Fig. 5: Equivalent Plant and Controller

As illustrated in Fig. 2, the LTI plant $P=N M^{-1}$ and LTI controller $C=V U^{-1}$ communicate with each other through cascaded two-port networks involving nonlinear perturbations. In particular, one can characterize the inputoutput pairs in the graph of $P$ as

$$
\left[\begin{array}{l}
u \\
y
\end{array}\right]=\left[\begin{array}{l}
M \\
N
\end{array}\right] x
$$

where $x \in \mathcal{H}_{2}$.

Consider the transmission type representation of the twoport networks $\left\{\boldsymbol{N}_{k}\right\}_{k=1}^{l}$. If the $k$-th stage of the network admits a stable nonlinear uncertainty $\boldsymbol{\Delta}_{k}$, then the transmission matrix is given as $\boldsymbol{T}_{k}=\boldsymbol{I}+\boldsymbol{\Delta}_{k}$. Signals in Fig. 5 have the following relations:

$$
\begin{aligned}
& {\left[\begin{array}{l}
u_{k} \\
y_{k}
\end{array}\right]=\left(\prod_{j=1}^{k} \boldsymbol{T}_{k+1-j}\right)\left[\begin{array}{l}
u \\
y
\end{array}\right]=\left(\prod_{j=1}^{k}\left(\boldsymbol{I}+\boldsymbol{\Delta}_{k+1-j}\right)\right)\left[\begin{array}{l}
u \\
y
\end{array}\right],} \\
& {\left[\begin{array}{c}
v_{k} \\
w_{k}
\end{array}\right]=\left(\prod_{j=k+1}^{l} \boldsymbol{T}_{j}^{-1}\right)\left[\begin{array}{c}
v \\
w
\end{array}\right]=\left(\prod_{j=k+1}^{l}\left(\boldsymbol{I}+\boldsymbol{\Delta}_{j}\right)^{-1}\right)\left[\begin{array}{c}
v \\
w
\end{array}\right] .}
\end{aligned}
$$

If we view $P$ together with $\left\{\boldsymbol{N}_{j}\right\}_{j=1}^{k}$ as an equivalent plant $\boldsymbol{P}_{k}^{e}$ with uncertainties $\left\{\boldsymbol{\Delta}_{j}\right\}_{j=1}^{k}$, then the graph of $\boldsymbol{P}_{k}^{e}$ is given by

$$
\mathcal{G}_{\boldsymbol{P}_{k}^{e}}=\left(\prod_{j=1}^{k}\left(\boldsymbol{I}+\boldsymbol{\Delta}_{k+1-j}\right)\right) \mathcal{G}_{P} .
$$

Similarly, if we view $C$ together with $\left\{\boldsymbol{N}_{j}\right\}_{j=k+1}^{l}$ as an equivalent controller $\boldsymbol{C}_{k}^{e}$ with uncertainties $\left\{\boldsymbol{\Delta}_{j}\right\}_{j=k+1}^{l}$, then the graph of $C_{k}^{e}$ is

$$
\mathcal{G}_{C_{k}^{e}}^{\prime}=\left(\prod_{j=k+1}^{l}\left(\boldsymbol{I}+\boldsymbol{\Delta}_{j}\right)^{-1}\right) \mathcal{G}_{C}^{\prime} .
$$

For convenience, we regard $k=0$ as the situation when $P$ is isolated from the two-port networks and $k=l$ when $C$ is isolated.

\section{Robust Stability Condition}

With the equivalent plant and controller representations derived aforehand, next we extend the definition on the stability of the two-port NCS in [10] to the nonlinear case.

As shown in Fig. 5, we denote the $k$-th input pair as $I_{k}:=$ $\left[p_{k}, q_{k}\right]^{T}$, the $k$-th output pair as $O_{k}:=\left[u_{k}, w_{k}\right]^{T}$ and the set of all outputs as $O:=\left[u_{1}, w_{1}, u_{2}, w_{2}, \ldots, u_{l}, w_{l}\right]^{T}$. By the feedback well-posedness assumption, the map from input $I_{k}$ to output $O$ exists and we denote it as $\boldsymbol{A}_{k}: I_{k} \in \mathcal{H}_{2} \mapsto$ $O \in \mathcal{H}_{2}$.

Definition 5. The two-port NCS in Fig. 5 is said to be stable if the operator $\boldsymbol{A}_{k}$ is finite-gain stable for every $k=0,1, \ldots, l$.

The following proposition further simplifies the stability condition.

Proposition 3. The two-port NCS is finite-gain stable if and only if the equivalent closed-loop system $\left[\boldsymbol{P}_{k}^{e}, \boldsymbol{C}_{k}^{e}\right]$ is finitegain stable for every $k=0,1, \ldots, l$.

Proof. Necessity holds trivially. Below we show sufficiency.

Let $\left[\boldsymbol{P}_{k}^{e}, \boldsymbol{C}_{k}^{e}\right]$ be finite-gain stable, and thus $\boldsymbol{F}_{\boldsymbol{P}_{k}^{e}, \boldsymbol{C}_{\boldsymbol{k}}^{e}}^{-1}$ is stable. As $\|\boldsymbol{\Delta}\|<1$ by hypothesis, both $\boldsymbol{I}+\boldsymbol{\Delta}_{j}$ and $(\boldsymbol{I}+$ $\left.\boldsymbol{\Delta}_{j}\right)^{-1}$ are stable. Hence the composite map of $\boldsymbol{F}_{\boldsymbol{P}_{\boldsymbol{k}}^{e}, \boldsymbol{C}_{\boldsymbol{k}}^{e}}^{-1}$ and $\boldsymbol{I}+\boldsymbol{\Delta}_{j}$ or $\left(\boldsymbol{I}+\boldsymbol{\Delta}_{j}\right)^{-1}$ is stable, which implies the stability of $\boldsymbol{A}_{k}$ for all $k=0,1, \ldots l$.

With the stability definition at hand, we present next the main robust stability theorem involving nonlinear perturbations in a two-port NCS.

In the following we assume that every closed-loop system $[\boldsymbol{P}, \boldsymbol{C}]$ is well-posed and $\boldsymbol{F}_{\boldsymbol{P}, \boldsymbol{C}}$ is surjective. Hence from Proposition 1 , the stability of $[\boldsymbol{P}, \boldsymbol{C}]$ is equivalent to the finite-gain stability of $\Pi_{\mathcal{G}_{P} / / \mathcal{G}_{C}^{\prime}}$. Let nominal LTI closed-loop system $[P, C]$ be stable.

Theorem 2. The two-port NCS is finite-gain stable for all $\left\{\boldsymbol{\Delta}_{k}\right\}_{k=1}^{l}$ subject to $\left\|\boldsymbol{\Delta}_{k}\right\| \leq r_{k}$ if and only if

$$
\sum_{k=1}^{l} \arcsin r_{k}<\arcsin b_{P, C} .
$$

From the above theorem, we know the stability margin $b_{P, C}$ is the same as that in a standard closed-loop system with "gap" uncertainties [11], [12], [21], hence the synthesis problem of a two-port NCS can be solved by an $\mathcal{H}_{\infty}$ optimization. In addition, the synthesis is irrelevant to detailed requirements of communication channels between the plant and controller, such as the number of two-port connections and how the uncertainty bounds are distributed among all the channels, which provide more flexibility on the selection of the communication channels.

Before proceeding to the proof of Theorem 2, we introduce a useful lemma.

Lemma 1. Given $r_{1}, r_{2} \in(0,1)$ and a closed conelike neighborhood $\mathcal{M} \subset \mathcal{H}_{2}$, it holds that

$$
\mathcal{S}\left(\mathcal{S}\left(\mathcal{M}, r_{1}\right), r_{2}\right) \subset \mathcal{S}\left(\mathcal{M}, \sin \left(\arcsin r_{1}+\arcsin r_{2}\right)\right) .
$$


Proof. Let $\mathcal{M}_{1}=\mathcal{S}\left(\mathcal{M}, r_{1}\right)$. Let $m \in \mathcal{M}, m_{1} \in \mathcal{M}_{1}$ and $m_{2} \in \mathcal{S}\left(\mathcal{S}\left(\mathcal{M}, r_{1}\right), r_{2}\right)$. Then we have

$$
\theta\left(m_{2}, m\right) \leq \theta\left(m_{2}, m_{1}\right)+\theta\left(m_{1}, m\right) .
$$

Particularly, take $\bar{m}_{1} \in \arg \min _{m_{1} \in \mathcal{M}_{1}} \theta\left(m_{2}, m_{1}\right)$. Then inequality (8) implies that

$$
\begin{aligned}
\theta\left(m_{2}, m\right) & \leq \theta\left(m_{2}, \bar{m}_{1}\right)+\theta\left(\bar{m}_{1}, m\right) \\
& \leq \arcsin r_{2}+\theta\left(\bar{m}_{1}, m\right) .
\end{aligned}
$$

Taking infimum at the both sides brings about that

$$
\begin{aligned}
\inf _{m \in \mathcal{M}} \theta\left(m_{2}, m\right) & \leq \arcsin r_{2}+\inf _{m \in \mathcal{M}} \theta\left(\bar{m}_{1}, m\right) \\
& \leq \arcsin r_{1}+\arcsin r_{2} .
\end{aligned}
$$

Hence, $m_{2} \in \mathcal{S}\left(\mathcal{M}, \sin \left(\arcsin r_{1}+\arcsin r_{2}\right)\right)$, which completes the proof.

The above lemma characterizes the inclusion relations of conelike sets. The proof of Theorem 2 is given next.

Proof of Theorem 2. The necessity follows from the "arcsin" theorem in [10] for LTI systems by noting that that the linear two-port neighborhood $\mathcal{N}\left(\mathcal{G}_{P}, r\right)$ is contained in the conelike set $\mathcal{S}\left(\mathcal{G}_{P}, r\right)$.

Next we prove the sufficiency. Assume we are at the $k$-th stage of equivalent closed-loop system as shown in Fig. 5 . Let $\mathcal{M}=\mathcal{G}_{\boldsymbol{P}}$ and $\mathcal{M}_{j}^{e}=\mathcal{G}_{P_{j}^{e}}, j=1,2, \ldots, l$. Then

$$
\mathcal{M}_{j}^{e}=\boldsymbol{T} \mathcal{M}_{j-1}^{e}=\left(\boldsymbol{I}+\boldsymbol{\Delta}_{j}\right) \mathcal{M}_{j-1}^{e}
$$

with $\left\|\boldsymbol{\Delta}_{j}\right\| \leq r_{j}$. Let $n \in \mathcal{M}_{j}^{e} \backslash\{0\}$, there exists an $m_{1} \in$ $\mathcal{M}_{j-1}^{e}$ such that $n=\left(\boldsymbol{I}+\boldsymbol{\Delta}_{j}\right) m_{1}$. Hence we have

$$
\inf _{0 \neq m \in \mathcal{M}_{j-1}^{e}} \frac{\|n-m\|_{2}}{\|m\|_{2}} \leq \frac{\left\|\boldsymbol{\Delta}_{j} m_{1}\right\|_{2}}{\left\|m_{1}\right\|_{2}} \leq\left\|\boldsymbol{\Delta}_{j}\right\| \leq r_{j} .
$$

As a result, $\mathcal{M}_{j}^{e} \subset \mathcal{S}\left(\mathcal{M}_{j-1}^{e}, r_{j}\right), j=2,3, \ldots, k$.

From Lemma 1 and by induction, we have

$$
\mathcal{M}_{k}^{e} \subset \mathcal{S}\left(\mathcal{M}_{k-1}^{e}, r_{k}\right) \subset \cdots \subset \mathcal{S}\left(\mathcal{M}, \sin \left(\sum_{j=1}^{k} \arcsin r_{j}\right)\right) \text {. }
$$

Likewise, for the controller part, let $\mathcal{N}=\mathcal{G}_{C}^{\prime}$ and $\mathcal{N}_{j}^{e}=$ $\mathcal{G}_{C_{j}^{e}}^{\prime}$. Then

$$
\mathcal{N}_{j-1}^{e}=\boldsymbol{T}^{-1} \mathcal{N}_{j}^{e}=\left(\boldsymbol{I}+\boldsymbol{\Delta}_{j}\right)^{-1} \mathcal{N}_{j}^{e}
$$

with $\left\|\boldsymbol{\Delta}_{j}\right\| \leq r_{j}$. Given any $n \in \mathcal{N}_{j-1}^{e} \backslash\{0\}$, there exists an $m_{1} \in \mathcal{N}_{j}^{e}$ such that $n=\left(\boldsymbol{I}+\boldsymbol{\Delta}_{j}\right)^{-1} m_{1}$. Hence we have

$$
\inf _{0 \neq m \in \mathcal{N}_{j}^{e}} \frac{\|n-m\|_{2}}{\|n\|_{2}} \leq \frac{\left\|\boldsymbol{\Delta}_{j} n\right\|_{2}}{\|n\|_{2}} \leq\left\|\boldsymbol{\Delta}_{j}\right\| \leq r_{j} .
$$

By Property 3 , we have

$$
\mathcal{N}_{j-1}^{e} \subset \tilde{\mathcal{S}}\left(\mathcal{N}_{j}^{e}, r_{j}\right)=\mathcal{S}\left(\mathcal{N}_{j}^{e}, r_{j}\right), j=k+1, \ldots, l .
$$

Hence by the same arguments as above, we have

$$
\mathcal{N}_{k}^{e} \subset \mathcal{S}\left(\mathcal{N}, \sin \left(\sum_{j=k+1}^{l} \arcsin r_{j}\right)\right)
$$

Therefore, from the stability condition (7) and Theorem 1. we know $\left[\boldsymbol{P}_{k}^{e}, \boldsymbol{C}_{k}^{e}\right]$ is stable for every $k=0,1, \ldots, l$. Combining this with Proposition 3 , we obtain the finite-gain stability of the two-port NCS.

\section{Scalability of the Stability Condition}

When we are faced with a large-scale network with many relays and connections, a particular communication link between a plant and a controller may involve many cascaded two-port channels. As the topology of an NCS changes, we need to confirm whether the new two-port communication link is "healthy" enough to keep the NCS robustly stable. Revaluating the whole network from the beginning may be impractical due to the limitations on computational resources or responding time. In the following, we show this problem can be solved in the two-port NCS by defining the stability residue properly.

For a two-port NCS with an LTI plant $P$ and LTI controller $C$ under nonlinear perturbations on its communication channels, define its stability residue as

$$
R_{P, C}\left(r_{1}, \ldots, r_{l}\right):=\arcsin b_{P, C}-\sum_{k=1}^{l} \arcsin r_{k},
$$

which is subsequently written as $R_{P, C}$ without ambiguity. It follows from Theorem 2 that the two-port NCS is stable for all stable uncertainties $\left\{\boldsymbol{\Delta}_{k}\right\}_{k=1}^{l}$ subject to $\left\|\boldsymbol{\Delta}_{k}\right\| \leq r_{k}$ if and only if $R_{P, C}\left(r_{1}, \ldots, r_{l}\right)>0$. It is no doubt that the larger $R_{P, C}$ is, the more robustly stable the NCS will be.

When some new two-port connections are added or some old ones are modified, checking whether the resulting NCS remains robustly stable becomes necessary. For this purpose, one only needs to update the stability residue and check its feasibility.

- When a new connection $\boldsymbol{T}_{\text {new }}=\boldsymbol{I}+\boldsymbol{\Delta}_{\text {new }}$ satisfying $\left\|\boldsymbol{\Delta}_{\text {new }}\right\| \leq r_{\text {new }}$ is added, let

$$
R_{P, C}^{\text {new }} \leftarrow R_{P, C}-\arcsin r_{\text {new }}
$$

- When an old connection $\boldsymbol{T}_{\text {old }}=\boldsymbol{I}+\boldsymbol{\Delta}_{\text {old }}$ with $\left\|\boldsymbol{\Delta}_{\text {old }}\right\| \leq$ $r_{\text {old }}$ is changed to $\boldsymbol{T}_{\text {new }}=\boldsymbol{I}+\boldsymbol{\Delta}_{\text {new }}$ satisfying $\left\|\boldsymbol{\Delta}_{\text {new }}\right\| \leq$ $r_{\text {new }}$, let

$$
R_{P, C}^{\text {new }} \leftarrow R_{P, C}-\arcsin r_{\text {new }}+\arcsin r_{\text {old }} .
$$

It follows from Theorem 2 and Equation (9) that the new NCS will be robustly stable if and only if $R_{P, C}^{\text {new }}>0$ after sequentially updating $R_{P, C}$ with respect to all the changes. In other words, the stability condition given in Theorem 1 is scalable as the network size is enlarged.

\section{CONCLUSion}

We investigate networked robust stabilization problem concerning LTI systems perturbed by nonlinear uncertainties. A special conelike uncertainty set is studied, which bridges the techniques of handling linear subspaces to those of handling nonlinear uncertainties in cascaded two-port networks. A necessary and sufficient stability condition is given in the form of an "arcsin" inequality, which is scalable when the size of the network is enlarged. As far as control synthesis is concerned, the problem can be solved through an $\mathcal{H}_{\infty}$ optimization of the closed-loop stability margin. 


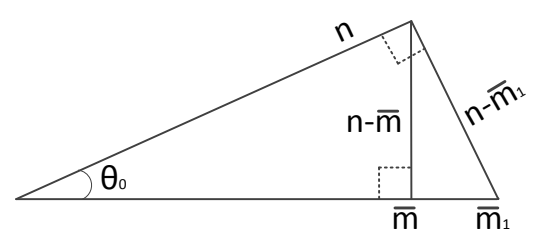

Fig. 6: Illustration for Proof of Property 3

\section{APPENDIX I}

Proofs of Properties 1, 2, ANd 3

Proof. From the closedness of the conelike set $\mathcal{M} \subset \mathcal{H}_{2}$, we can replace "inf" with "min" in the definition of $\tilde{\mathcal{S}}(\mathcal{M}, r)$ and $\mathcal{S}(\mathcal{M}, r)$. Next we prove the properties in turns.

For Property 1, it suffices to show for $n \in \mathcal{S}(\mathcal{M}, r)$, it holds $\alpha n \in \mathcal{S}(\mathcal{M}, r)$ for every $\alpha \in \mathbb{R}$. Using the definition, we have

$$
\inf _{0 \neq m \in \mathcal{M}} \frac{\|\alpha n-m\|}{\|m\|}=\inf _{0 \neq \alpha m \in \mathcal{M}} \frac{\|\alpha n-\alpha m\|}{\|\alpha m\|} \leq r,
$$

which establishes Property 1 .

For Property 2, let $n \in \mathcal{S}(\mathcal{M}, r)$. It follows that

$$
\bar{m} \in \underset{m \in \mathcal{M}}{\arg \min } \frac{\|n-m\|_{2}}{\|m\|_{2}}
$$

satisfies $n-\bar{m} \perp n$, whereby $\sin \theta(\bar{m}, n)=\frac{\|n-\bar{m}\|_{2}}{\|\bar{m}\|_{2}} \leq r$. Consequently, $\mathcal{S}(\mathcal{M}, r) \subset\left\{n \in \mathcal{H}_{2}: \min _{m \in \mathcal{M}} \theta(m, n) \leq\right.$ $\arcsin r\} \cup\{0\}$. On the other hand, let $n$ belongs to the latter set. From Property 1. we can find $\bar{m} \in \mathcal{S}(\mathcal{M}, r)$ such that $\theta(\bar{m}, n)=\arcsin r$ and $n-\bar{m} \perp n$, which implies that

$$
\frac{\|n-\bar{m}\|_{2}}{\|\bar{m}\|_{2}}=\sin \theta(\bar{m}, n) \leq r .
$$

This completes the proof for Property 2

For Property 3 , given $n \in \tilde{\mathcal{S}}(\mathcal{M}, r)$, consider

$$
\bar{m} \in \arg \min _{m \in \mathcal{M}} \frac{\|n-m\|_{2}}{\|n\|_{2}} .
$$

It follows that $n-\bar{m} \perp \bar{m}$. Denote the acute angle between $n$ and $\bar{m}$ as $\theta_{0}(\leq \arcsin r)$. In the hyperplane determined by $\bar{m}$ and $n$, as shown in Fig. 6, we can extend $\bar{m}$ to $\bar{m}_{1} \in \mathcal{M}$ along $\bar{m}$ such that $n-\bar{m}_{1} \perp n$, which is guaranteed by Property 1 . This implies that

$$
\min _{0 \neq m \in \mathcal{M}} \frac{\|n-m\|_{2}}{\|m\|_{2}} \leq \frac{\left\|n-\bar{m}_{1}\right\|_{2}}{\left\|\bar{m}_{1}\right\|_{2}}=\sin \theta_{0} \leq r .
$$

Consequently, $\tilde{\mathcal{S}}(\mathcal{M}, r) \subset \mathcal{S}(\mathcal{M}, r)$.

On the other hand, given $n \in \mathcal{S}(\mathcal{M}, r)$ and consider

$$
\bar{m} \in \arg \min _{0 \neq m \in \mathcal{M}} \frac{\|n-m\|_{2}}{\|m\|_{2}},
$$

one can argue likewise that $\mathcal{S}(\mathcal{M}, r) \subset \tilde{\mathcal{S}}(\mathcal{M}, r)$, which completes the proof.

\section{ProOF OF PROPOSITION2}

Proof. We prove both parts by contradiction. The proposition holds trivially when $[P, C]$ is unstable, thus it suffices to prove the case when $[P, C]$ is stable. For brevity, define $\mathcal{M}:=\mathcal{G}_{P}, \mathcal{N}:=\mathcal{G}_{C}^{\prime}, \mathcal{M}_{1}:=\mathcal{G}_{P_{1}}$ and $\mathcal{N}_{1}:=\mathcal{G}_{C_{1}}^{\prime}$.

\section{Sufficiency:}

Let $\mathcal{G}_{\boldsymbol{P}_{1}} \subset \mathcal{S}\left(\mathcal{G}_{P}, r_{p}\right), \mathcal{G}_{\boldsymbol{C}_{1}}^{\prime} \subset \mathcal{S}\left(\mathcal{G}_{C}^{\prime}, r_{c}\right)$ and $\left[\boldsymbol{P}_{1}, \boldsymbol{C}_{1}\right]$ be unstable. It follows that $\Pi_{\mathcal{M}_{1} / / \mathcal{N}_{1}}$ is unbounded. That is to say, there exists a sequence $\left\{\omega_{k}\right\}_{k=1}^{\infty} \subset \mathcal{H}_{2} \backslash\{0\}$, such that:

- $\left\|\omega_{k}\right\|_{2} \nearrow \infty$

- $\lim _{k \rightarrow \infty} \frac{\left\|\Pi_{\mathcal{M}_{1} / / \mathcal{N}_{1}} \omega_{k}\right\|_{2}}{\left\|\omega_{k}\right\|_{2}}=\infty$.

By the surjectivity of $\boldsymbol{F}_{\boldsymbol{P}_{1}, \boldsymbol{C}_{1}}$, we know that

$$
\omega_{k}=\Pi_{\mathcal{M}_{1} / / \mathcal{N}_{1}} \omega_{k}+\Pi_{\mathcal{N}_{1} / / \mathcal{M}_{1}} \omega_{k}=: m_{k}+n_{k} .
$$

Hence, $\alpha_{k}:=\frac{\left\|\omega_{k}\right\|_{2}}{\left\|m_{k}\right\|_{2}} \rightarrow 0$ as $k \rightarrow \infty$. From Definition 3 we know $m_{k}, n_{k} \in \mathcal{H}_{2} \backslash\{0\}$, and thus the angle between them can be computed as

$$
\theta\left(m_{k}, n_{k}\right)=\arccos \left|\frac{\left\langle m_{k}, n_{k}\right\rangle}{\left\|m_{k}\right\|_{2}\left\|n_{k}\right\|_{2}}\right| .
$$

Consequently,

$$
\begin{aligned}
\cos \theta\left(m_{k}, n_{k}\right) & =\left|\frac{\left\langle m_{k}, n_{k}\right\rangle}{\left\|m_{k}\right\|_{2}\left\|n_{k}\right\|_{2}}\right| \\
& \geq\left|\frac{\left\langle m_{k}, n_{k}\right\rangle}{\left\|m_{k}\right\|_{2}\left(\left\|m_{k}\right\|_{2}+\left\|\omega_{k}\right\|_{2}\right)}\right| \\
& =\frac{1}{1+\alpha_{k}}\left(1-\frac{\left|\left\langle m_{k}, \omega_{k}\right\rangle\right|}{\left\|m_{k}\right\|_{2}^{2}}\right) \\
& \geq \frac{1}{1+\alpha_{k}}\left(1-\frac{\left\|m_{k}\right\|_{2}\left\|\omega_{k}\right\|_{2}}{\left\|m_{k}\right\|_{2}^{2}}\right) \\
& =\frac{1-\alpha_{k}}{1+\alpha_{k}} \rightarrow 1 \quad \text { as } k \rightarrow \infty .
\end{aligned}
$$

Hence $\theta\left(m_{k}, n_{k}\right) \rightarrow 0$. Since $\mathcal{G}_{\boldsymbol{P}_{1}}$ and $\mathcal{G}_{\boldsymbol{C}_{1}}^{\prime}$ are closed sets, it follows that $\mathcal{G}_{\boldsymbol{P}_{1}} \cap \mathcal{G}_{\boldsymbol{C}_{1}}^{\prime} \neq\{0\}$ and therefore $\mathcal{S}\left(\mathcal{M}, r_{p}\right) \cap$ $\mathcal{S}\left(\mathcal{N}, r_{c}\right) \neq\{0\}$, which leads to a contradiction.

\section{Necessity:}

Assume there exists a nonzero $u$ satisfying $u \in$ $\mathcal{S}\left(\mathcal{M}, r_{p}\right) \cap \mathcal{S}\left(\mathcal{N}, r_{c}\right)$. From Property 1 we know $\{\alpha u: \alpha \in$ $\mathbb{R}\} \subset \mathcal{S}\left(\mathcal{M}, r_{p}\right) \cap \mathcal{S}\left(\mathcal{N}, r_{c}\right)$. Construct two scalar sequences $\left\{\alpha_{k}\right\}_{k=1}^{\infty}$ and $\left\{\beta_{k}\right\}_{k=1}^{\infty} \subset \mathbb{R}$ such that

- $\left|\alpha_{k}\right|,\left|\beta_{k}\right|$ and $\left|\frac{\alpha_{k}}{\beta_{k}}\right| \nearrow \infty$;

- $\beta_{k}=\alpha_{t}+\left(\beta_{l}-\alpha_{l}\right)$ if and only if $k=t=l$.

Furthermore, construct two graphs $\mathcal{M}_{1}:=\mathcal{G}_{\boldsymbol{P}_{1}} \subset$ $\mathcal{S}\left(\mathcal{M}, r_{p}\right), \mathcal{N}_{1}:=\mathcal{G}_{C_{1}}^{\prime} \subset \mathcal{S}\left(\mathcal{N}, r_{c}\right)$, such that $\left\{\alpha_{k} u\right\} \subset \mathcal{M}_{1}$, $\left\{\left(\beta_{k}-\alpha_{k}\right) u\right\} \subset \mathcal{N}_{1}$ and $\boldsymbol{F}_{\boldsymbol{P}_{1}, \boldsymbol{C}_{1}}$ is surjective. Hence, for any $\omega_{k}=\beta_{k} u \in \mathcal{H}_{2}$, we have the decomposition

$$
\omega_{k}=\beta_{k} u=\alpha_{k} u+\left(\beta_{k}-\alpha_{k}\right) u:=m_{k}+n_{k} .
$$

Moreover,

$$
\lim _{k \rightarrow \infty} \frac{\left\|m_{k}\right\|_{2}}{\left\|\omega_{k}\right\|_{2}}=\lim _{k \rightarrow \infty}\left|\frac{\alpha_{k}}{\beta_{k}}\right|=\infty .
$$

It follows directly that $\Pi_{\mathcal{M}_{1} / / \mathcal{N}_{1}}$ is unbounded, i.e. $\left[\boldsymbol{P}_{1}, \boldsymbol{C}_{1}\right]$ is unstable, which leads to a contradiction. 


\section{REFERENCES}

[1] W. Zhang, M. S. Branicky, and S. M. Phillips, "Stability of networked control systems," IEEE Contr. Syst., vol. 21, no. 1, pp. 84-99, 2001.

[2] R. J. Anderson and M. W. Spong, "Bilateral control of teleoperators with time delay," IEEE Trans. Automat. Contr, vol. 34, no. 5, pp. 494-501, 1989.

[3] F. Alagoz and G. Gur, "Energy efficiency and satellite networking: A holistic overview," Proc. IEEE, vol. 99, no. 11, pp. 1954-1979, Nov. 2011.

[4] A. A. Kumar S., K. Ovsthus, and L. M. Kristensen., "An industrial perspective on wireless sensor networks - a survey of requirements, protocols, and challenges," IEEE Commun. Surveys Tut., vol. 16, no. 3, pp. 1391-1412, 3rd Quarter 2014.

[5] B. Wu, J. Chen, J. Wu, and M. Cardei, "A survey of attacks and countermeasures in mobile ad hoc networks," Wireless Netw. Security, pp. 103-135, 2007.

[6] U. Bakshi and A. Bakshi, Network Analysis. Pune, India: Technical Publications, 2009.

[7] H. Kimura, Chain-Scattering Approach to $\mathcal{H}_{\infty}$ Control. New York: Springer Science \& Business Media, 1996.

[8] S. Z. Khong and M. Cantoni, "Reconciling $\mu$-gap metric and IQC based robust stability analysis," IEEE Trans. Automat. Contr., vol. 58, no. 8, pp. 2090-2095, 2013.

[9] G. Gu and L. Qiu, "A two-port approach to networked feedback stabilization," in Proc. 50th IEEE Conf. on Decision and Contr. and European Contr. Conf. (CDC-ECC), pp. 2387-2392, Dec. 2011.

[10] D. Zhao and L. Qiu, "Networked robust stabilization with simultaneous uncertainties in plant, controller and communication channels," in Proc. 55th IEEE Conf. on Decision and Contr. (CDC), pp. 2376-2381, Dec. 2016.

[11] K. Zhou and J. C. Doyle, Essentials of Robust Control. Upper Saddle River, NJ: Prentice Hall, 1998.

[12] L. Qiu and E. Davison, "Feedback stability under simultaneous gap metric uncertainties in plant and controller," Syst. Contr. Lett., vol. 18, no. 1, pp. 9-22, 1992.

[13] M. Siami, T. Hayakawa, H. Ishii, and K. Tsumura, "Adaptive quantized control for linear uncertain systems over channels subject to packet loss," in Proc. 49th IEEE Conf. on Decision and Contr. (CDC), pp. 4655-4660, Dec. 2010.

[14] L. Su and G. Chesi, "Robust stability analysis and synthesis for uncertain discrete-time networked control systems over fading channels," IEEE Trans. Automat. Contr., vol. 62, no. 4, pp. 1966-1971, 2017.

[15] J. C. Willems, The Analysis of Feedback Systems. Clinton, Massachusetts: The M.I.T Press, 1971.

[16] M. Vidyasagar, Nonlinear System Analysis. Englewood Cliffs, NJ: Prentice Hall, 1993.

[17] S. Z. Khong, M. Cantoni, and J. H. Manton, "A gap metric perspective of well-posedness for nonlinear feedback interconnections," Australian Contr. Conf. (AUCC), pp. 224-229, Nov 2013.

[18] J. C. Doyle, T. T. Georgiou, and M. C. Smith, "The parallel projection operators of a nonlinear feedback system," Syst. Contr. Lett., vol. 20, no. 2, pp. $79-85,1993$.

[19] G. Vinnicombe, Uncertainty and Feedback: $\mathcal{H}_{\infty}$ loop-shaping and the $\nu$-gap metric. Singapore: World Scientific, 2000.

[20] G. Zames, "On the input-output stability of time-varying nonlinear feedback systems-Part II: Conditions involving circles in the frequency plane and sector nonlinearities," IEEE Trans. Automat. Contr., vol. 11, no. 3, pp. 465-476, 1966.

[21] L. Qiu and E. J. Davison, "Pointwise gap metrics on transfer matrices," IEEE Trans. Automat. Contr., vol. 37, no. 6, pp. 741-758, 1992. 